\title{
Resolver problemas: una estrategia para el aprendizaje de la termodinámica*
}

\section{Problem Solving: A Strategy in Learning Thermodynamics}

\section{José Joaquín García García Edilma Rentería Rodríguez}

Referencia para citar este artículo: GARCIA G., J. J. y RENTERÍA RODRIGUEZ, E. (2013). "Resolver problemas: una estrategia para el aprendizaje de la termodinámica". En: Revista Guillermo de Ockham 11(2). pp. 117-134.

\section{Resumen}

En este trabajo se analiza la influencia de una estrategia didáctica basada en la resolución de problemas que usa los procedimientos propios de la modelización experimental en el aprendizaje de los conceptos básicos de la termodinámica. El estudio tuvo un carácter cuasi experimental con un grupo control, un grupo experimental y la aplicación de un post-test. El test aplicado contaba con dieciocho ítems distribuidos en nueve factores referidos a la comprensión de los conceptos básicos de la termodinámica. Dicho test presentó un alfa $(\alpha)$ de Crombach de 0.61 considerado aceptable. El análisis de los datos se hizo a través de dos procedimientos: en primer lugar, comparando los porcentajes de respuestas correctas obtenidos por cada uno de los dos grupos en los ítems contemplados en la prueba. En segundo lugar, realizando una prueba t de Student de comparación de las medias obtenidas por el grupo experimental y el grupo control, en el test sobre comprensión de los conceptos termodinámicos. Los resultados de ambas comparaciones, permitieron concluir que la estrategia didáctica alternativa es mejor para lograr el aprendizaje conceptual en los estudiantes que la estrategia didáctica convencional.

Palabras clave: modelización, experimentación, resolución de problemas, aprendizaje, termodinámica.

\section{Abstract}

This research work analyzes the influence of a didactic strategy based on problem solving and uses the model and experimental proceedings in the learning

- Fecha de recepción del artículo: 31-04-2013 • Fecha de aceptación: 31-07-2013

JOSÉ JOAQUíN GARCÍA GARCíA. Doctor en Didáctica de las Ciencias Experimentales por la Universidad de Granada. Docente Titular de la Universidad de Antioquia, Medellín, Colombia. Correo electrónico: yocolombiano@yahoo.com.mx. EDILMA RENTERÍA RODRÍGUEZ Magíster en Educación énfasis Ciencias Experimentales por la Universidad de Antioquia. Docente catedrática de la Universidad de Antioquia, Medellín, Colombia. Correo electrónico: edilmarenteria@yahoo.es.

* Este artículo de investigación científica y tecnológica es producto del proyecto "Interacciones complejas entre los procesos de modelización y resolución de problemas con el uso de múltiples representaciones científicas externas (semióticas) en la educación en ciencias experimentales" adelantado por el Grupo de Investigación "Innovaciencia" de la Facultad de Educación, Universidad de Antioquia. Fecha de inicio: enero de 2007. Fecha de finalización: abril de 2011. 
of the basic concepts of thermodynamics. This was a quasi-experimental study with an experimental and a control group. A post-testing was used. The test had 18 items and 9 factors about the understanding of basic concepts of thermodynamics. This test had a Cronbach's alpha $(\alpha)$ of 0.61 . The data analysis was done through two procedures. First, the percent of correct answers obtained by each groups were compared with the items included in the test. Secondly, a Student's t-Test was used to compare the averages obtained by the experimental and control group in the test on understanding basic concepts of thermodynamics. From the results of both comparisons, it can be concluded that the alternative strategy is better to achieve the conceptual learning than a conventional didactic strategy.

Keywords: modeling, experimentation, solving problem, learning, thermodynamics.

\section{Introducción}

En este artículo se presenta parte de los resultados de una investigación de carácter científico y tecnológico en el área de la didáctica de las ciencias experimentales, en la cual se pretendió determinar el efecto de la aplicación de una estrategia didáctica basada en la resolución de problemas abiertos y contextualizados que incorpora procedimientos de modelización, en el aprendizaje conceptual de la termodinámica de estudiantes de último grado de bachillerato. De acuerdo con lo anterior, en primer lugar se expondrán los principios teóricos básicos del enfoque de resolución de problemas en la enseñanza de las ciencias y del proceso de modelización asociado a dicha resolución. En segundo lugar, se plantearán los elementos más sobresalientes de la estrategia didáctica utilizada. En tercer lugar, se presentarán los resultados obtenidos tanto por el grupo control como por el grupo experimental. En cuarto y último lugar, se propondrán algunas conclusiones basadas en el análisis de los resultados obtenidos en la investigación.

\section{La resolución de problemas en la educación científica}

En la didáctica tradicional, la resolución de problemas no interesantes con un exceso de ope- rativismo y un proceso de resolución superficial, favorece el afianzamiento de los preconceptos en los estudiantes y desfavorece el análisis y el cuestionamiento (Gil, Martínez y Senent, 1988). Además sucede, ya que en dicha metodología prevalecen las certezas y no hay dudas ni soluciones alternativas, las cuales son más bien fijas e inmediatas, originadas en el sentido común y sin relación con las disciplinas. Por el contrario, un modelo didáctico en el que se pueda aplicar una metodología científica para resolver situaciones problema, que implique construir hipótesis, diseñar experimentos, ejecutarlos y analizar sus resultados, genera un aprendizaje significativo y un cambio conceptual en los estudiantes que los posibilita para detectar y corregir sus errores y los incita a tener una actitud positiva hacia el problema y el aprendizaje.

Por otra parte, la resolución de problemas cualitativos en el aula mejora la comprensión conceptual y evita que se busquen datos e incógnitas para operar sin comprender los principios y los conceptos teóricos relacionados con el problema (García, 2003). Es decir, se posibilita el análisis de la situación física y su representación y reformulación para establecer las relaciones significativas entre sus conceptos claves en el diseño de estrategias de resolución. Así mismo, ayuda a darle significado al problema y a comprender la conexión entre las relaciones matemáticas (ecuaciones) y sus correlatos físicos. Además, la construcción del conocimiento conceptual y procedimental mediante la resolución de situaciones problema es una elaboración autónoma en la que se aprende a pensar. Dicha construcción implica la comprensión de los modelos y principios presentes en los enunciados de los problemas para asignarles significado. Esto presupone relacionar dichos modelos con las experiencias y conocimientos previos y la transferencia de dichos conceptos para planificar estrategias de resolución de los problemas. Dichas situaciones problema propuestas en el aula deben ser creativas y relacionadas con los conceptos científicos y para su elaboración los docentes podrían utilizar recursos de la historia de las ciencias, de su epistemología y de los contextos en los cuales se utilizan los conceptos que se van a enseñar así como su cotidianidad e implicaciones sociales, económicas y tecnológicas.

El aprendizaje conceptual obtenido mediante la resolución de situaciones problema, se puede 
evaluar determinando la capacidad de trasferencia de los conceptos a situaciones y contextos diferentes para explicar y predecir el comportamiento de sistemas naturales, sociales o culturales.

\section{La modelización en las ciencias}

Para establecer las relaciones entre la teoría y los fenómenos estudiados, se utilizan como articuladores a los modelos científicos. Así, un modelo científico representa tanto una parte de la teoría científica como del fenómeno en estudio (Moreira, Greca, y Rodríguez, 2002). Es decir, es la parte aplicativa de una teoría (Lorenzano, 2008), pero no es una copia de la realidad que se desea estudiar. De esta forma, los modelos se caracterizan por ser idealizaciones de la realidad (Giere, 1992; Concari. 2001 y Adúriz, Izquierdo, 2008); o sea, no son una réplica verdadera del mundo, sino que lo representan de forma válida bajo ciertas condiciones en las que se incluyen solo las variables que se desean estudiar; así, la producción de modelos es una actividad central de la ciencia (Bachelard, 1991; Islas y Pesa 2001). Por medio de dichos modelos los científicos manipulan los fenómenos y solucionan problemas (Islas y Pesa, 2001 y Adúriz e izquierdo, 2008).

Para la construcción de los modelos, se describen, bajo condiciones concretas, los sistemas y subsistemas se y especifican su estructura, disposición, conexiones y propiedades mediante representaciones ideales con el menor número de predicados y determinando cómo cambian en el tiempo y en el espacio, es decir, posibilitando la generalización y la predicción de su comportamiento (Harré 1967). Así mismo, si lo que se requiere es explicar el comportamiento del sistema, se sustituyen sus propiedades cualitativas por formas cuantitativas o se vinculan a otras propiedades que sean cuantificables y mensurables, a condición de que estas varíen con las propiedades cualitativas; así, los científicos al describir, explicar o predecir el comportamiento de un sistema mediante teorías y modelos, llevan a cabo actividades de modelización. Entre dichas actividades se encuentran plantear problemas, formular hipótesis, identificar los objetos del sistema, asignarles propiedades conceptuales, establecer relaciones relevantes entre las variables previamente establecidas, hacer inferencias, proponer prediccio- nes y construir diseños experimentales para probar las hipótesis. En esta investigación se propuso la aplicación de una estrategia didáctica para la resolución de problemas cualitativos, abiertos y contextualizados, y la modelización experimental para el aprendizaje de los conceptos básicos de la termodinámica (ver Anexo 1). En ella se plantean los siguientes pasos:

- Entender el problema; diferenciar lo inteligible de lo que no lo es.

- Identificar las condiciones de resolución que ofrece el problema.

- Construir hipótesis para resolverlo usando marcos teóricos.

- Diseñar un modelo experimental para contrastar las hipótesis, así:

- Establecer la información que se puede obtener del modelo físico.

- Identificar sus variables experimentales y extrañas; cómo medirlas y controlarlas.

- Representar las relaciones supuestas entre las variables.

- Construir el modelo físico y chequear su idoneidad.

- Experimentar con el modelo físico.

- Formular un modelo matemático sobre las relaciones determinadas experimentalmente entre las variables estudiadas, lo que requiere:

- Determinar su objetivo.

- Elaborar y ajustar un gráfico que relacione los datos de las variables.

- Enunciar verbalmente dicha relación y expresarla luego matemáticamente.

- Dar respuesta al problema planteado y contrastarla con la hipótesis.

\section{Metodología}

La metodología usada en esta investigación fue de carácter cuasi experimental. Se conformaron un grupo control y uno experimental y se aplicó una postprueba a ambos grupos una vez finalizado el empleo de una estrategia de enseñanza alternativa en el grupo experimental y la aplicación de una estratega didáctica de corte tradicional en el grupo control. Ambas estrategias se dirigieron a lograr 
el aprendizaje por parte de los estudiantes de los conceptos básicos de la termodinámica. Ambos grupos cursaban el undécimo grado y estaban conformados por veintinueve estudiantes con una edad promedio de 17.3 años que cursaban la asignatura Introducción a la Física en la Institución Pública San Luis Gonzaga del municipio de Copacabana (Antioquia, Colombia). El estudio se llevó a cabo en el primer semestre del 2009 durante siete semanas, tiempo durante el cual en el grupo control se implementó una estrategia didáctica convencional. Dicha estrategia se ejecutó de la manera que sigue. En primer lugar cada uno de los apartados correspondientes a la termodinámica básica fue explicado por la docente a sus estudiantes mediante una exposición magistral. En segundo lugar y luego de la explicación el profesor proponía a los estudiantes ejercicios (con lápiz y papel) de carácter algorítmico en los cuales el estudiante solo tenía que determinar que ecuaciones o las fórmulas matemáticas necesarias para su resolución. En tercer lugar se dejaban para hacer en casa ejercicios similares a los resueltos en clase con el objetivo de que se mecanicen las formas de resolución de los ejercicios.

Durante el mismo tiempo de siete semanas, se empleó en el grupo experimental una estrategia didáctica basada en la resolución de problemas usando la modelización experimental. Dicha estrategia comprendía los siguientes principios y actividades centrales. En primer lugar se enfrentaba a los estudiantes a situaciones problema cualitativas y contextualizadas cuya resolución requería más de la construcción de los conceptos básicos de la termodinámica que de la aplicación mecánica de fórmulas y ecuaciones. Los problemas, al ser cualitativos, reducían al mínimo los componentes matemáticos y hacían énfasis tanto en las relaciones existente entre variables como en la naturaleza de los conceptos implicados en el problema. Así mismo, al ser dichos problemas contextualizados aludían a situaciones como el comportamiento de los glaciales o el funcionamiento de un olla a presión; es decir, a fenómenos conocidos para los estudiantes y relacionados con su vida cotidiana y sus posibles intereses. En segundo lugar, para el tratamiento de los problemas propuestos se siguieron los pasos sugeridos en la última parte del apartado sobre la modelización y consignado en este artículo. Dicho tratamiento estuvo orientado por los procesos de modelización.

A ambo grupos se los evaluó usando el mismo test para medir el aprendizaje conceptual de principios, leyes y conceptos básicos de termodinámica, el cual indagaba sobre la comprensión de conceptos y principios termodinámicos básicos mediante dieciocho ítems distribuidos en nueve factores y un alfa $(\alpha)$ de Crombach de 0.61 (ver Anexo 2). La hipótesis propuesta fue la siguiente: "Una estrategia didáctica basada en la resolución de problemas y articulada a la modelización experimental, influye positivamente en el aprendizaje de los conceptos básicos de la termodinámica de estudiantes de undécimo grado". En cuanto a las variables tenidas en cuenta en este estudio, la variable manipulada fue la estrategia didáctica con dos valores: tradicional y alternativa, y la observada fue el aprendizaje de conceptos de la termodinámica con nueve factores.

Factor 1. Comprensión del concepto de temperatura (TE). La comprensión de la temperatura es un concepto métrico que mide el promedio de la energía cinética de las moléculas de un sistema. Se operativiza en el aspecto macroscópico con el indicador "comprensión de la propiedad intensiva de la temperatura", que precisa que esta no depende de la cantidad de masa del cuerpo.

Factor 2. Comprensión del concepto de calor (CA). El calor es un proceso por medio del cual se transfiere energía de un sistema a otro debido a la diferencia de temperatura entre estos. Para medir la comprensión de los estudiantes del concepto de calor se usan dos indicadores: establecer la diferencia entre calor y temperatura, y comprender el calor como energía en tránsito.

Factor 3. Comprensión del concepto de energía interna (EI). La energía interna es una función de estado de un sistema y está constituida por la suma de las energías cinética, potencial, vibratoria y rotacional de las moléculas de un cuerpo. Así, la energía interna de un cuerpo depende de la cantidad de materia de este y de su temperatura. Para evaluar este factor se utilizan como indicadores: comprender que la energía interna depende de la masa y la energía interna de la temperatura.

Factor 4. Comprensión del concepto de calor especifico (CE). El calor específico es la cantidad de transferencia de energía que requiere un de- 


\section{Tabla 1}

Factores de la prueba sobre comprensión conceptual de los principios y conceptos básicos de termodinámica.

\begin{tabular}{|c|c|}
\hline Factor (FAC) & $\begin{array}{l}\text { Indicadores } \\
\text { (IND) }\end{array}$ \\
\hline $\begin{array}{l}\text { 1. Comprensión del concepto de temperatura } \\
\text { (TE) }\end{array}$ & Propiedad intensiva. \\
\hline \multirow[t]{2}{*}{ 2. Comprensión del concepto de calor (CA) } & Diferencia calor de temperatura \\
\hline & Calor como energía en tránsito \\
\hline \multirow{2}{*}{$\begin{array}{l}\text { 3. Comprensión del concepto de energía interna } \\
\text { (EI) }\end{array}$} & La energía interna depende de la masa \\
\hline & La energía interna depende de la temperatura \\
\hline $\begin{array}{l}\text { 4. Comprensión del concepto de calor específico } \\
\text { (CE) }\end{array}$ & Concepto de calor específico \\
\hline \multirow{2}{*}{$\begin{array}{l}\text { 5. Comprensión del concepto de equilibrio } \\
\text { térmico (ET) }\end{array}$} & Estimación de la temperatura después del equilibrio térmico \\
\hline & Concepto de equilibrio térmico \\
\hline \multirow{2}{*}{$\begin{array}{l}\text { 6. Compresión del comportamiento de la } \\
\text { temperatura durante los cambios de fase (CF) } \\
\text { y después de estos }\end{array}$} & Temperatura después del cambio de fase \\
\hline & Temperatura durante el cambio de fase \\
\hline \multirow[t]{2}{*}{ 7. Comprensión del concepto de presión (PR) } & Relación presión/temperatura \\
\hline & Relación presión/volumen \\
\hline \multirow{2}{*}{$\begin{array}{l}\text { 8. Compresión de la primera ley de la termodi- } \\
\text { námica (PL) }\end{array}$} & Energía en procesos dinámicos \\
\hline & Conservación de la energía \\
\hline \multirow{4}{*}{$\begin{array}{l}\text { 9. Compresión de la segunda ley de la termodi- } \\
\text { námica (SL) }\end{array}$} & Dirección en la que fluye el calor \\
\hline & Degradación de la energía \\
\hline & Funcionamiento de las máquinas térmicas \\
\hline & Características de las máquinas ideales \\
\hline
\end{tabular}

terminado material (cada tipo) para cambiar su temperatura. Para medir este factor se indica el calor específico de algunos materiales para que el estudiante lo relacione con la cantidad de energía necesaria para variar su temperatura.

Factor 5. Comprensión del concepto de equilibrio térmico (ET). Según la ley cero de la termodinámica, si dos cuerpos están en equilibrio térmico con respecto a un tercero estos dos cuerpos se encuentran en equilibrio térmico entre sí. Para evaluar este factor los estudiantes deben estimar la temperatura que alcanzan dos cuerpos en contacto térmico que inicialmente están a diferentes temperaturas (mismo material y masa) después del equilibrio térmico y comprender el concepto de equilibrio térmico, determinando que la temperatura de dos cuerpos de diferentes materiales en contacto térmico con el ambiente es la misma.

Factor 6. Comprensión del comportamiento de la temperatura en cambios de fase y después de ellos $(C F)$. Durante el cambio de estado, un sistema permanece con temperatura constante aunque haya transferencia de energía y después del cambio de fase el sistema cambia su temperatura (Delgado, 2002). Para evaluar la comprensión de este factor los estudiantes deben determinar el comportamiento de la temperatura de varios cuerpos durante el cambio de fase y después de este debido a un proceso de transferencia de energía.

Factor 7. Comprensión del concepto presión (PR). Para evaluar la percepción de este concepto se utilizan dos indicadores: el entendimiento de la relación entre presión y temperatura al determinar la influencia que tiene la variación de la presión en la temperatura que alcanzan los cuerpos expuestos a una misma transferencia de energía, y la comprensión de la relación entre la presión y el volumen mediante la determinación de la variación de la presión del sistema al variar su volumen sin que haya transferencia de energía térmica.

Factor 8. Comprensión de la primera ley de la termodinámica (PL). La primera de ley termo- 
dinámica establece que la energía transferida de un sistema a otro debido a las interacciones termodinámicas se conserva. Es decir, que la energía no varía su magnitud al pasar de una forma a otra o de un sistema a otro. Para evaluar el etendimiento de esta ley se usan los siguientes indicadores: comprender que en los procesos dinámicos hay transferencia de energía debido al trabajo sin que haya transferencia de energía térmica, y captar que la magnitud de la energía en los procesos termodinámicos se conserva cuando un sistema está expuesto a transferencia de energía térmica.

Factor 9. Comprensión y aplicación de la segunda ley de la termodinámica (SL). De acuerdo con la segunda ley de la termodinámica "no es posible un proceso en el que el único resultado final sea la transferencia de calor desde una temperatura más baja a otra más alta”; es decir, no existe un ciclo térmico en el que se extraiga calor a temperatura constante y se convierta completamente en trabajo" (Gettys, Keller y Skove, 1991).

Para evaluar este factor se utilizan los siguientes indicadores: determinar que en una situación en la que hay transferencia de energía térmica el calor fluye espontáneamente desde la fuente que se encuentra a mayor temperatura hacia la fuente que está a menor temperatura; identificar la degradación de la energía en un gráfico que representa el funcionamiento de una máquina térmica; comprender el proceso de funcionamiento de las máquinas térmicas; y establecer las características de la máquina ideal.

\section{Resultados}

Las diferencia de 5.31 puntos entre los valores de la media global obtenidos por los grupos control (media de 6.20) y experimental (media de 11.51) en el test sobre el aprendizaje conceptual de la termodinámica es significativa: $\mathrm{t}=9.73 ; \mathrm{p}=0.000$. Es decir, la estrategia didáctica alternativa basada en la resolución de problemas usando modelización experimental sí influye positivamente en el aprendizaje de los conceptos básicos de la termodinámica en los estudiantes de undécimo grado. A continuación se presentan los resultados para cada factor en los dos grupos (ver Gráficos 1, 2 y 3).

Factor 1. Comprensión del concepto de temperatura. Aunque en ambos grupos un alto porcentaje de estudiantes (62\% y $97 \%$, respectivamente) comprende que la temperatura de un cuerpo no depende de su masa, este es mayor en el grupo experimental tal vez porque pudo comprobar experimentalmente la naturaleza intensiva de la temperatura al elaborar y probar modelos en lugar de aprenderlo memorísticamente para luego proceder a la resolución de ejercicios, tal como lo hizo el grupo control. Por otra parte, el que un porcentaje de ambos grupos considere aún a la temperatura como una propiedad extensiva, concuerda con los resultados de otros trabajos (De Berg, 2008).

Factor 2. Comprensión del concepto de calor. Este concepto es mejor aprendido por los estudiantes del grupo experimental que por los del grupo control (65\% y $45.5 \%$ de respuestas correctas, respectivamente). El $41 \%$ del grupo experimental

Gráfico 1

Distribución porcentual del grupo control según su dominio de los conceptos de la termodinámica

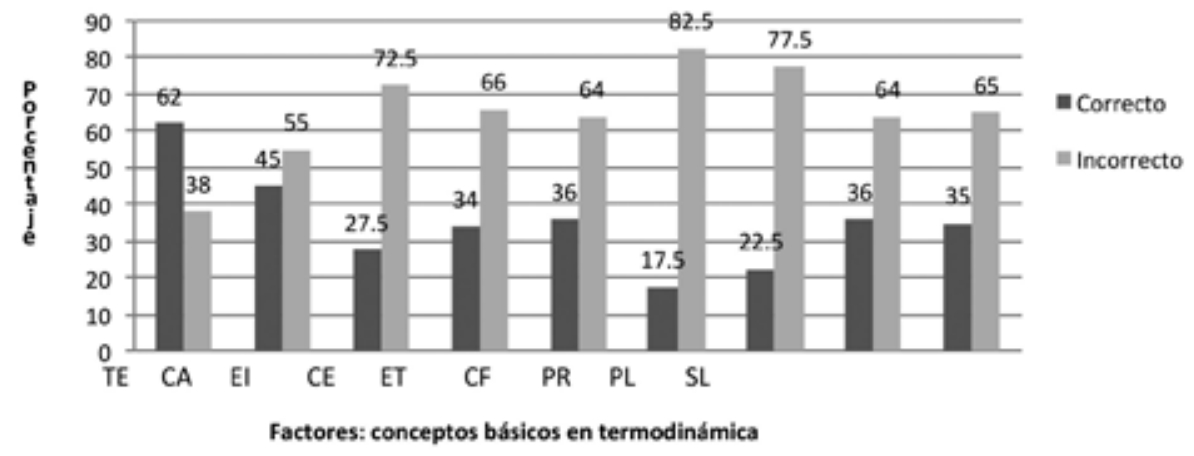

$122<$ Universidad de San Buenaventura, Cali - Colombia 
Gráfico 2

Distribución porcentual del grupo experimental según su dominio de los conceptos de la termodinámica

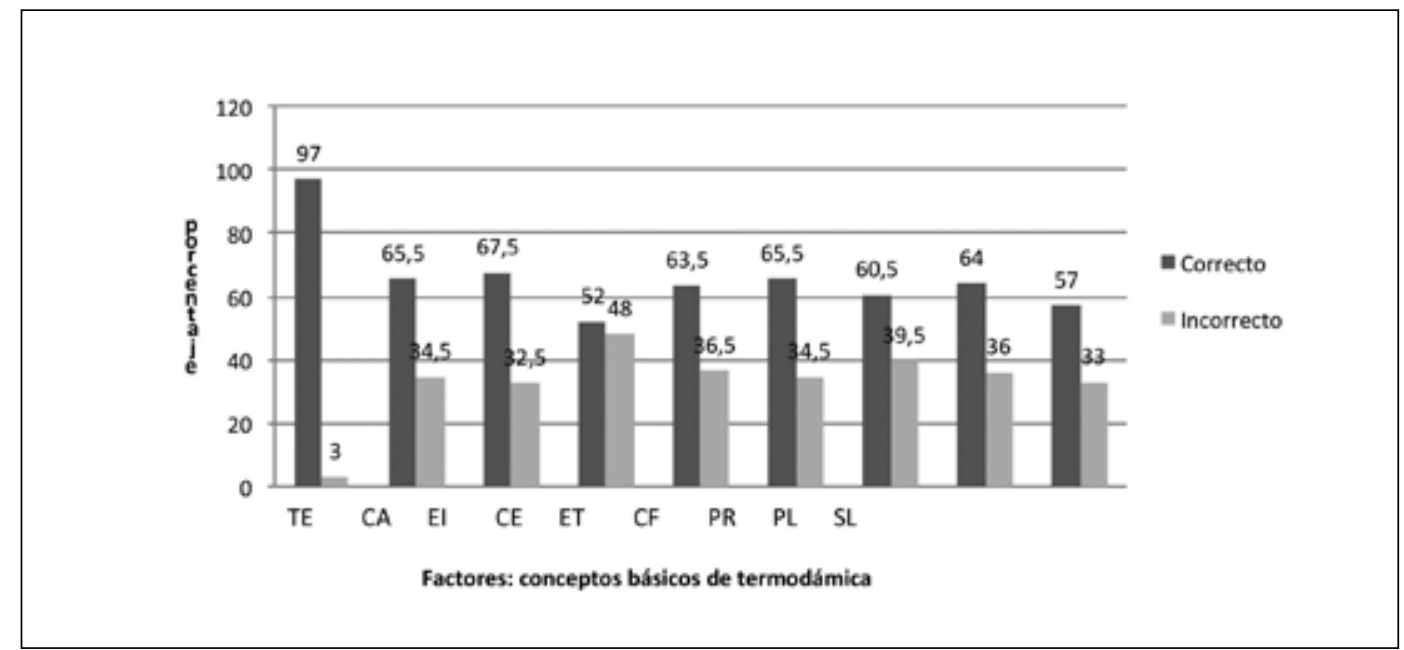

Gráfico 3

Distribución porcentual comparada de los grupos control y experimental según su dominio de los conceptos de la termodinámica

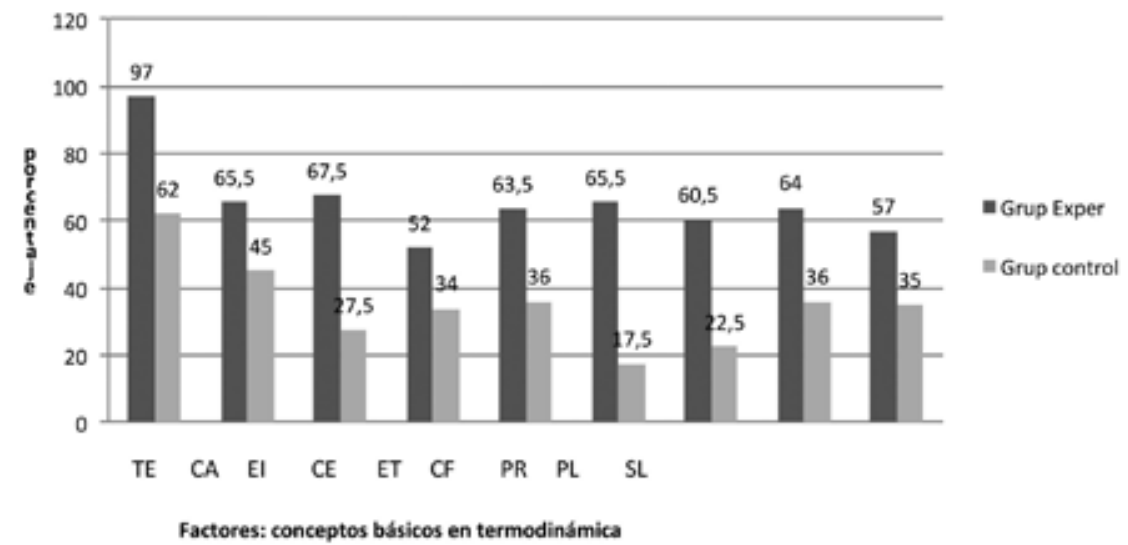

todavía concibe el calor como una sustancia que puede entrar y salir de un objeto, y un $26 \%$ de este lo considera como sinónimo de temperatura (a mayor temperatura mayor calor), creyendo con ello innecesario que dos cuerpos a diferentes temperaturas estén en contacto para que dicho calor se produzca. Esto muestra la persistencia de estas concepciones, lo que concuerda con los resultados de otras investigaciones (Delgado y otros, 2002; González, 2003; Furió, Solbes y Furió, 2007).

Factor 3. Comprensión del concepto de energía interna. Mientras solo el $17 \%$ del grupo experimental no acaba de comprender que la energía interna depende de la masa, este nivel de incom- prensión se eleva al $83 \%$ en el grupo control. Así mismo, el $48 \%$ del grupo experimental ni el $65 \%$ del grupo control no comprenden que el cambio de energía interna en un sistema depende de su temperatura. Estos resultados pueden deberse a que los problemas cualitativos resueltos por el grupo experimental estaban orientados a establecer la relación entre la cantidad de masa y la cantidad de energía interna de un sistema, como también a precisar la relación entre el cambio de temperatura y el cambio de energía interna.

Factor 4. Comprensión de concepto de calor especifico. Un $51 \%$ en el grupo experimental comprende el concepto de calor específico mientras solo un 
$34 \%$ del grupo control lo hace. La diferencia puede ser debida a que el grupo control solo calculaba el valor numérico del calor específico en el marco de ejercicios, mientras que el grupo experimental, al resolver problemas cualitativos orientados a fenómenos como la formación de corrientes de aire en la zonas costeras o a la determinación de temperatura de la arena con relación a la del agua en un día de sol, se enfocaba al establecimiento de la relación entre el tipo de material y la temperatura alcanzada por este en un proceso de transferencia de energía (Vázquez, 2004).

Factor 5. Comprensión del concepto de equilibrio térmico. Solo el $34 \%$ de los estudiantes del grupo control puede estimar correctamente la temperatura que alcanzan dos o más cuerpos después del equilibrio térmico (inicialmente a diferentes temperaturas), mientras que en el grupo experimental lo puede hacer el $66 \%$. Estas diferencias pueden deberse a que el grupo control calculó algorítmicamente la temperatura que alcanzan dos o más cuerpos después del equilibrio térmico, logrando con ello una comprensión mecanicista y superficial; mientras que el grupo experimental logró una comprensión conceptual y más profunda al resolver problemas mediante el diseño de modelos experimentales para determinar la temperatura alcanzada por dos sustancias después de juntarlas.

Estos resultados concuerdan con los de otras investigaciones en las que se manifiesta que muchos estudiantes consideran que la temperatura de una mezcla de sustancias cada una con diferente temperatura inicial, es igual a la suma de las temperaturas iniciales de las sustancias (Engels, 1982). Por otra parte, solo el $38 \%$ de los estudiantes del grupo control comprende que si dos cuerpos están en equilibrio térmico con el medio ambiente estos están en equilibrio térmico entre sí; mientras que en el grupo experimental este porcentaje es del $72 \%$. Esto puede deberse a que los estudiantes del grupo control, al no tener la oportunidad de diseñar experimentos acerca de la temperatura alcanzada por diferentes materiales en contacto térmico con el medio ambiente, siguen explicando este fenómeno a partir de la sensación térmica al tocar diferentes materiales que están en contacto térmico con el medio ambiente y por ello creen que dichos cuerpos están a diferentes temperaturas (Cervantes, De la Torre, Verdejo, Trejo, Córdoba y Flores, 2001).
Factor 6. Comprensión del comportamiento de la temperatura durante los cambios de fase y luego de estos. Mientras el $84 \%$ del grupo experimental comprende el comportamiento de la temperatura en un cuerpo después de un cambio de fase, solo el $14 \%$ del grupo control lo comprende. Al parecer, la mera explicación sobre el comportamiento de la temperatura después de un cambio de fase no es suficiente para comprender dicho proceso; por el contrario, la resolución de problemas cualitativos en los cuales se deben elaborar e interpretar gráficos para caracterizar dicho comportamiento sí es efectiva. Otros estudios con resultados similares muestran, por ejemplo, que los estudiantes creen que la temperatura del hielo únicamente puede ser de $0^{\circ} \mathrm{C}$ (Delgado y otros, 2002). Los resultados también muestran que solo el $21 \%$ de los estudiantes del grupo control comprenden el comportamiento de la temperatura de los cuerpos durante el cambio de fase, mientras que este mismo fenómeno es comprendido por el $79 \%$ del grupo experimental. Las diferencias encontradas pueden originarse en la resolución por parte del grupo experimental de problemas cualitativos específicamente orientados a la comprensión del comportamiento de la temperatura de los cuerpos durante un cambio de fase. Por otra parte, el hecho de que en ambos grupos haya estudiantes que consideren que durante el cambio de fase de un cuerpo la temperatura de este no varía, puede deberse a que su creencia de que al seguir el proceso de transferencia de energía la temperatura debe variar (Bañas, Mellado y Ruiz, 2003).

Factor 7. Comprensión del concepto de presión. Solo el $7 \%$ del grupo control establece correctamente la relación entre la presión y la temperatura, mientras que este porcentaje es del $55 \%$ en el grupo experimental. Así mismo, mientras solo el $38 \%$ del grupo control establece la relación entre la presión y el volumen, el $66 \%$ del grupo experimental logra establecerla. Esto puede interpretarse como ineficacia didáctica de procedimientos consistentes en la resolución de ejercicios en los cuales se calcula la temperatura o el volumen de un sistema en relación con su presión mediante la aplicación matemática de las formalizaciones, y por su parte, como un mayor éxito didáctico de las actividades experimentales en la que se pueden verificar y analizar dichas relaciones (Flores, Alons, Delgado y Trejo, 2005). Estas dificultades concep- 
tuales impedirían dilucidar termodinámicamente las diferencias en la temperatura de ebullición de un mismo líquido en diferentes lugares o la mejora de la eficacia y el rendimiento de los motores de alta presión.

Factor 8. Comprensión de la primera ley de la termodinámica. El $66 \%$ del grupo experimental comprende que en los procesos dinámicos hay transferencia de energía, mientras que en el grupo control solo lo comprende el $38 \%$. Igualmente, el $62 \%$ del grupo experimental entiende que la energía en un proceso termodinámico se conserva, mientras que en el grupo control solo lo hace un $34 \%$. Es decir, si se conocen dos de las tres variables, el cálculo del calor, del trabajo o del cambio de energía interna en un sistema en el marco de ejercicios numéricos no permite llegar a comprender la naturaleza de la transferencia energética generada por el proceso termodinámico, lo cual requiere la determinación experimental de dichas variable; por ejemplo, a través de la determinación de la variación de la temperatura debida al trabajo realizado para evidenciar el cambio de energía interna. Estos resultados coinciden con otra investigaciones en las cuales se reporta que muchas veces los estudiantes no consideran el trabajo como un proceso de transferencia de energía y asumen que el cambio de energía interna en el sistema es ocasionado solo por la transferencia de calor (Loverude, Kautz y Heron, 2002).

Factor 9. Comprensión y aplicación de la segunda ley de la termodinámica. El $55 \%$ del grupo experimental comprende que en el funcionamiento de las máquinas térmicas todo el calor no se convierte en trabajo (la energía se degrada), mientras que solo el $34 \%$ del grupo control sí lo comprende. Estos porcentajes de respuestas correctas se repiten cuando se determinan la dirección en la cual fluye el calor y se ponen en contacto dos sistemas a diferentes temperaturas. Además, el $66 \%$ del grupo experimental comprende el funcionamiento de las máquinas térmicas, es decir, cómo se transforma el calor en trabajo, mientras que en el grupo control solo lo comprende el $31 \%$. Por último, el funcionamiento de las máquinas ideales solo es comprendido por el $49 \%$ del grupo control y por el $55 \%$ del grupo experimental. Estos resultados hablan a favor de la resolución de problemas prácticos, como el de establecer las condiciones para que un vehículo sea lo menos contaminante posible y dejan en entredicho la ejecución de actividades como el cálculo teórico de la eficacia de una máquina térmica, cuando de lo que se trata es de hacer reflexionar más a los estudiantes sobre sus concepciones acerca de los fenómenos termodinámicos y lograr su comprensión.

\section{Conclusiones}

Los resultados obtenidos apuntan a la conclusión de que la resolución de problemas abiertos y prácticos que abordan cuestiones sobre el papel de la termodinámica en la tecnología, la naturaleza y la sociedad, posibilita un mejor aprendizaje de los conceptos termodinámicos que una estrategia convencional basada en una metodología expositiva. Ello se explicaría dado que este tipo de problemas probablemente estimulan su resolución al permitir la participación activa, la emisión de juicios, la reflexión y la construcción de conocimiento en todo el proceso, al acotarlos, emitir hipótesis y diseñar modelos experimentales para manipular las variables y comprobar dichas hipótesis en un proceso no lineal, de análisis, evaluación y diseño. De igual manera, el análisis de los resultados experimentales permite inferir que esta estrategia didáctica alternativa influye positivamente y con mayor intensidad en el aprendizaje de los conceptos de temperatura, calor, energía interna, equilibrio térmico, energía y presión y en la comprensión del comportamiento de la temperatura durante los cambios de fase y después de estos así como de la primera ley de la termodinámica. Con menor intensidad influye en el aprendizaje de los conceptos de calor específico y de la segunda ley de la termodinámica, tal vez por su mayor complejidad y por requerir de la comprensión de los primeros. 


\section{Bibliografía}

- ADÚRIZ-BRAVO Agustín y IZQUIERDO, Merce (2008). Un modelo de modelos científicos para la enseñanza de las ciencias naturales. En: Revista Electrónica de Investigación en Educación en Ciencias 4(1). pp. 40-49.

- BACHELARD, Gaston (1991). Critic of Science and the Imagination. Reino Unido: Ed. Routledge Taylor y Francis Group.

- BAÑAS, Carlos; MELLADO, Vicente y RUIZ, Constantino (2003). Las ideas alternativas del alumnado de primer ciclo de Educación Secundaria Obligatoria sobre la conservación de la energía el calor y la temperatura. En: Campo Abierto 24. pp. 99-126.

- CERVANTES, Leticia; DE LA TORRE, Natalia; VERDEJO, Adriana; TREJO, Luis Miguel; CÓRDOBA, José Luis y FLORES, Fernando (2001). El concepto de calor en termodinámica y su enseñanza. En: Memorias del XVI congreso nacional de termodinámica, México D.F. Universidad de Colima y SMT.

- CONCARI, Sonia Beatriz (2001). Las teorías y modelos en la explicación científica: implicancias para la enseñanza de las ciencias. En: Ciência y Educação (Bauru) 7(1). pp. 85-94.

- CRAWFORD, Barbara A. y CULLIN, Michael. J. (2004). Supporting prospective teachers' conceptions of modeling in science. En: International Journal of Science Education 26 (11). pp. 1379-1401.

- DE BERG, Kelvin Charles (2008). The concepts of heat and temperature: the problem of determining the content for the construction of an historical case study which is sensitive to nature of science issues and teaching-learning issues. En: Science y Education 17 (Springer Netherlands). pp. 75-114.

- DELGADO, Teresa; FLORES, Susana y TREJO, Luis Miguel| (2002). Calor latente: propuesta de enseñanza del concepto. En: Memorias del XVII congreso nacional de termodinámica. México D.F. Universidad de Colima y SMT.

- ENGEL, Elizabeth (1982). The development of understanding of selected aspects of pressure, heat and evolution in pupils aged between 12-16 years. Unpublished Ph.D thesis, University of Leeds, Leeds UK.

- FLORES, Susana; ALONSO, Guadalupe; DELGADO, Teresa y TREJOS, Luis. Miguel (2005). Innovación en el laboratorio de termodinámica. En: Enseñanza de las ciencias, Número Extra. IV Congreso.

- FURIÓ-GÓMEZ. Carles; SOLBES, Jordi y FURIÓ-MAS, Carlos José (2007). La historia del primer principio de la termodinámica y sus implicaciones didácticas. En: Revista Eureka sobre Enseñanza y Divulgación de las Ciencias 4 (3). pp. 461-475.

- GARCÍA, José Joaquín (2003). Didáctica de las ciencias. Resolución de problemas y desarrollo de la creatividad. Bogotá: Editorial Magisterio.

- GETTYS, Edward; KELLER, Frederick y SKOVE, Malcom (1991). Física clásica y moderna. Madrid: Editorial McGrawn - Hill.

- GIERE, Ronald N. (1992). La explicación de la ciencia. Un acercamiento cognoscitivo México: Consejo Nacional de Ciencia y Tecnología.

- GIL, Daniel; MARTÍNEZ-TORREGROSA, Joaquín y SENENT, F. (1988). El fracaso en la resolución de problemas de física: una investigación orientada por nuevos supuestos. En: Enseñanza de las ciencias, 6(2). pp.131-164.

- GONZÁLEZ, Arnaldo (2003). Calor y trabajo en la enseñanza de la termodinámica. En Revista cubana de fisica 20 (2). (Sociedad Cubana de Física). pp. 1011-1026.

- HARRÉ, Rom (1967). Introducción a la lógica de las ciencias. Barcelona: Editorial Labor.

- HARRISON, Allan G. y TREAGUST, David F. (2000). A typology of school science models. En: Inernational Journal of Science Education 22(9). pp. 1011-1026.

$126<$ Universidad de San Buenaventura, Cali - Colombia 
- ISLAS, Estela Maris y PESA, Martha A. (2003). ¿Qué rol asignan los profesores de física de nivel medio a los modelos científicos y a las actividades de modelado? En: Enseñanza de las ciencias Número Extra (Barcelona: Universitat Autònoma de Barcelona). pp.57-66.

- LORENZANO, Pablo (2008). Inconmensurabilidad teórica y comparabilidad empírica: el caso de la genética clásica. En: Documento interno. Universidad Nacional de Quilmes.

- LOVERUDE, Michael E.; KAUTZ, Christian y HERON, Paula (2002). Student Understanding of the first law of thermodynamics: relating work to the adiabatic compression of an ideal gas. En: American journal of physics 70(2). (American Association of Physics Teachers with American Institute of Physics). pp. 137-148. Doi: $10.1119 / 1.1417532$

- MOREIRA, Marco Antonio; GRECA, Ilena María y RODRÍGUEZ, Martha Luz (2002). Modelos mentales y modelos conceptuales en la enseñanza y aprendizaje de las ciencias. En: Revista Brasileira de Pesquisa em Educação em Ciências 2(3). (Associação Brasileira de Pesquisa em Educação em Ciências). pp. 36-56.

- VÁZQUEZ, Carlos (2004). Reflexiones y ejemplos de situaciones didácticas para una adecuada contextualización de los contenidos científicos en el proceso de enseñanza. En: Revista Eureka sobre enseñanza y divulgación de las ciencias 1(3). pp. 214-223. 


\section{Anexo 1.}

\section{Algunos problemas abiertos usados en la estrategia didáctica}

\section{¿Cómo influye el calor específico de la superficie de la Tierra en la formación de las corrientes de aire en las zonas costeras?}

Carolina vive en Nuquí (departamento del Chocó, Colombia) a orillas del océano Pacífico. Ella desea aprovechar las corrientes de aire para generar energía eléctrica a fin de abastecer las necesidades de su vivienda. ¿Qué características deben tener las superficies terrestres para que se produzcan las corrientes de aire? Según puntos de referencia como las costas y el mar, ¿en qué sentido sopla el viento en las noches? ¿En qué sentido sopla el viento en el día? ¿Por qué lo hace de esta manera? Durante un día de sol, ¿cómo crees que es la temperatura de la arena en relación con el agua durante el día? ¿Cómo crees que es la temperatura de la arena con relación al agua en la noche?

Diseńa un modelo experimental en el que se puedan generar corrientes de aire haciendo uso del calor específico de los materiales.

\section{¿Depende de su masa la temperatura de un cuerpo?}

El glacial de Groenlandia, al igual que otros glaciales del mundo, se está descongelando. El agua producto del descongelamiento es vertida al océano Glacial Ártico que a su vez se comunica con el océano Atlántico y el océano Pacífico. ¿De qué manera afecta el descongelamiento del glacial de Groenlandia a un país como Colombia? (su área es 1.7 millones de kilómetros cuadrados por tres kilómetros de profundidad). ¿Puede alcanzar la mezcla de las dos aguas una temperatura de $5^{\circ} \mathrm{C}$ si la temperatura del agua del glacial está inicialmente a $2^{\circ} \mathrm{C}$ y la temperatura del Océano Ártico está a $8^{\circ} \mathrm{C}$ ? ¿Qué condiciones debe haber para que la mezcla tenga esas temperaturas? ¿¿Puede alcanzar dicha mezcla una temperatura de $10^{\circ} \mathrm{C}$ ? ¿Qué condiciones debe haber para alcanzar dicha temperatura? ¿De qué variables depende la temperatura que alcanza una mezcla de dos líquidos que se encuentran a diferentes temperaturas?

Diseña un experimento que te permita dar respuestas a las preguntas planteadas.

\section{¿Cómo varía la temperatura en los cambios de estado del agua?}

El agua tiene un proceso cíclico: se evapora, se condensa y regresa al suelo, bien sea en forma líquida o sólida. Dicho proceso en gran medida depende de la cantidad de rayos del sol que reciba la Tierra. ¿Qué cambios puede sufrir el ciclo del agua debido al calentamiento del planeta? ¿Cómo afectan al hombre los cambios en el ciclo del agua? ¿Qué cambios de fase sufre el agua debido a la influencia del sol? ¿Qué cambio de temperatura tiene el agua durante el proceso de evaporación? ¿Cuál es la temperatura del punto de ebullición del agua en Copacabana? Durante el proceso de condensación, ¿cómo varía la temperatura del agua? Durante el proceso de congelamiento, ¿cómo varia la temperatura del agua? ¿Cuál es la temperatura de fusión del agua?

Diseña un experimento que permita evidenciar los cambios de fase que tiene el agua durante el ciclo natural y determinar el comportamiento de la temperatura en dichos cambios de fase. 


\section{¿Qué cambios de energía se experimenta en las máquinas de vapor durante su funcionamiento?}

Una empresa tiene máquinas de vapor que funcionan con la energía calórica que emerge de las profundidades de la Tierra. Las máquinas tiene la estructura que a continuación se describe y su funcionamiento sigue el siguiente proceso. El vapor a altas temperaturas es transportado desde el fondo de la Tierra a la superficie a través de un tubo el cual desemboca en un cilindro que tiene un émbolo. Cuando se abre la llave A y se cierra la llave B el émbolo se desplaza hacia arriba y la máquina realiza un trabajo. Cuando se cierra la llave A y se abre la llave B el émbolo vuelve a su punto de partida para iniciar el proceso de nuevo. ¿Qué aparatos conoces que utilizan para su funcionamiento energía en forma de calor? ¿Qué ventajas crees que ofrece la utilización de fuentes de energía geotérmica a la humanidad y cuáles desventajas conlleva? Representa mediante un dibujo la situación. Lleva a cabo un experimento mediante el cual puedas establecer la relación entre la energía suministrada en forma de calor a una máquina, el cambio de energía interna del motor y el trabajo hecho por la máquina. Formula una ecuación matemática que represente dicha situación. ¿Cómo cambia la energía interna de la máquina de vapor si se le suministra energía pero esta no realiza trabajo? Representa la ecuación de dicha situación. 


\section{Anexo 2}

\section{Test para medir el aprendizaje conceptual de principios, leyes y conceptos básicos de termodinámica}

Nombre del estudiante

grado

En el cuadernillo marque la opción que considere correcta y justifique la respuesta.

1. Se toman dos pedazos de hielo de un glacial. El pedazo de hielo $\mathrm{A}$ es diez veces más grande que el pedazo B. Si simultáneamente se mide la temperatura con un termómetro a los dos pedazos de hielos se puede decir:

a. La temperatura del pedazo de hielo $\mathrm{A}$ es mayor que la del pedazo $\mathrm{B}$.

b. La temperatura del pedazo de hielo $B$ es mayor que la del pedazo $A$.

c. El pedazo de hielo A tiene la misma temperatura del pedazo de hielo B.

d. La temperatura de una sustancia depende de la cantidad de su masa.

2. Hay dos cubículos aislados térmicamente. En el cubículo A hay una taza de chocolate a $100^{\circ} \mathrm{C}$ y en el cubículo $\mathrm{B}$ hay un helado de chocolate a $-2^{\circ} \mathrm{C}$. Comparando los dos sistemas podemos decir que:

a. El vaso con chocolate contiene mayor cantidad de calor que el helado de chocolate.

b. El vaso con chocolate contiene menor cantidad de calor que el helado.

c. Los dos sistemas contienen la misma cantidad de calor.

d. El helado de chocolate y la taza de chocolate no contienen calor.

3. En una noche de invierno, si una persona cubre su cuerpo con una cobija de lana la temperatura de este se mantiene más alta que la del medio ambiente. Dicho fenómeno se debe a que:

a. La cobija contiene calor y se lo transmite al cuerpo de la persona.

b. La cobija impide que entre el frío del medio ambiente al cuerpo de la persona.

c. La cobija impide que fluya la energía calórica de la persona al medio ambiente.

d. La persona le suministra calor a la cobija, manteniendo esta la temperatura alta.

4. En un centro recreacional hay dos piscinas. La de los adultos es dos veces más grande que la de los nińos. Si el agua de ambas está a $25^{\circ} \mathrm{C}$ de temperatura, se puede decir que:

a. La piscina de los niños tiene menor energía interna que la piscina de los adultos.

b. Ambas piscinas tienen la misma cantidad de energía interna.

c. La piscina de los niños tiene mayor cantidad de energía interna que la piscina de los adultos.

d. La energía interna de un sistema no depende su masa.

5. En una cafetería venden un vaso de leche caliente al mismo precio que un vaso de leche fría. Si la cantidad de leche caliente es igual a la cantidad de leche fría, se puede decir que:

a. En el vaso de leche caliente hay mayor energía interna que en el vaso de leche fría.

b. En el vaso de leche fría hay mayor cantidad de energía interna que en el vaso de leche cliente. 
c. Ambos vasos de leche tienen la misma cantidad de energía interna, pues tienen igual masa.

d. La energía interna no la contienen los objetos, es el proceso de transferencia de energía.

6. Para refrigerar su carro, Adriana tiene la opción de utilizar el líquido A o el líquido B. Si el calor específico del líquido $B$ es mayor que el calor específico del líquido $A$, se puede decir que:

a. El líquido $\mathrm{A}$ es mejor refrigerador que el líquido $\mathrm{B}$, porque para aumentar la temperatura el líquido $\mathrm{A}$ necesita más transferencia de energía que el líquido $\mathrm{B}$.

b. El líquido $\mathrm{B}$ es mejor refrigerador que el líquido $\mathrm{A}$, porque para aumentar la temperatura el líquido $\mathrm{B}$ necesita más transferencia de energía que el líquido $\mathrm{A}$.

c. Los dos líquidos refrigeran el motor de igual manera, porque ambos necesitan de la misma transferencia de energía para aumentar la temperatura.

d. El líquido $B$ es mejor refrigerador que el líquido A, porque para aumentar la temperatura el líquido $\mathrm{B}$ necesita menos transferencia de energía que el líquido $\mathrm{A}$.

7. Se ponen tres objetos a diferentes temperaturas en contacto pero aislados térmicamente, como se muestra en la siguiente figura. Las temperaturas de los objetos se relacionan de la siguiente manera : $\mathrm{TC}>\mathrm{TB}>\mathrm{TA}$ ( $\mathrm{TC}$ es mayor que TB y está es mayor que $\mathrm{TA})$.

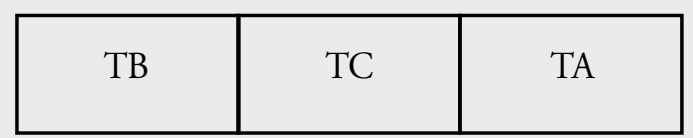

Después del equilibrio térmico del sistema la temperatura a la que queda el sistema es:

a. Mayor que de TC.

b. Menor de TA.

c. Mayor de TB y menor de TA.

d. Menor de TC y mayor de TA.

8. En una época de invierno en la cual la temperatura se ha mantenido constate por mucho tiempo, la madre de Santiago le dice: "Ponte las sandalias de plástico porque el piso de cemento está más frío que las sandalias". De dicha afirmación se puede afirmar que:

a. Es correcta, porque la temperatura del piso es menor que la temperatura de las sandalias.

b. Es incorrecta, porque el piso y las sandalias están a la misma temperatura debido al equilibrio térmico.

c. Es correcta, porque el piso está a mayor temperatura que las sandalias.

d. Es incorrecto, porque la temperatura del piso es menor que la de las sandalias.

9. Diego está haciendo ejerció y como consecuencia su cuerpo se sobrecalienta. Suda y su sudor se evapora. La evaporación del sudor de Diego permite que:

a. La temperatura de su cuerpo baje.

b. La temperatura de su cuerpo aumente.

c. La temperatura de su cuerpo se mantenga constante.

d. No conozco la respuesta.

10. Una olla de café inicia su proceso de ebullición a $95^{\circ} \mathrm{C}$. Si al café se le suministra energía en forma de calor por un determinado tiempo, se puede decir que:

a. Cuanto más hierva el café más aumenta la temperatura.

b. La temperatura del café durante el hervor se mantiene constante.

c. La temperatura del café disminuye a medida que este hierve.

d. No conozco la respuesta. 
11. El tiempo de cocción de un determinado alimento depende de la tempera a la que se ponga a cocer dicho alimento. En la Tierra hay lugares con presión atmosférica alta y con presión atmosférica baja. ¿Qué personas economizan energía para cocer sus alimentos de acuerdo con su posición?

a. Las personas que están ubicadas en lugares con presión atmosférica alta.

b. Las personas que están ubicadas en lugares con presión atmosférica baja.

c. La presión atmosférica no influye en la velocidad de cocción de los alimentos.

d. No conozco la respuesta.

12. Un cilindro que tiene un émbolo, al ser comprimido pasa de la posición 1 a la 2.

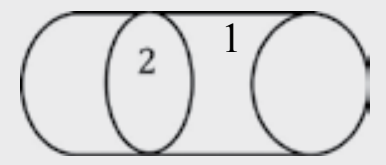

La gráfica que mejor representa la relación entre presión y volumen es:

a.

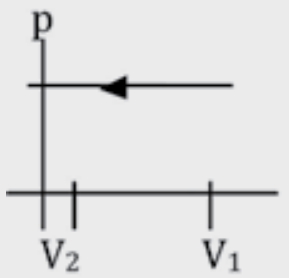

b.

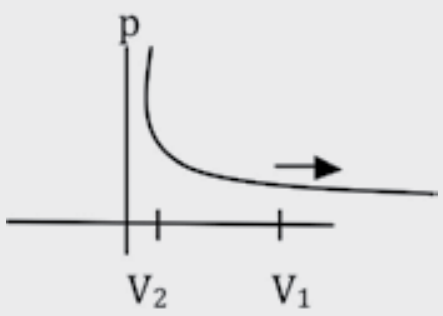

c.

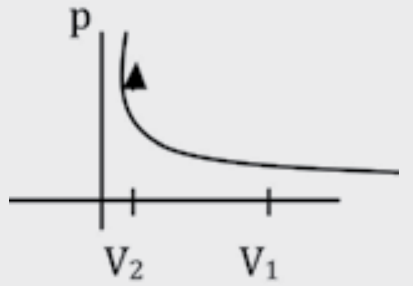

d.

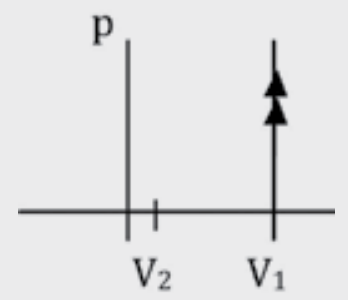

13. Un neumático es inflado con una bomba para bicicleta. Cuando se comprime el aire se evidencia un aumento en la temperatura de la bomba. Si la esta está aislada térmicamente, se puede decir que:

a. La energía interna de la bomba permanece constante.

b. El cambio de la energía interna de la bomba es igual al calor suministrado.

c. El cambio en la energía interna de la bomba es igual al trabajo hecho.

d. El cambio en la energía interna es igual al aumento en la temperatura.

14. Una olla a presión tiene el orificio de la válvula tapada. Esta es colocada sobre una estufa que le suministra energía térmica (calor). La ecuación que mejor representa la energía que interviene en dicho proceso es:
a. $\Delta \mathrm{U}=-\mathrm{Q}+\mathrm{W}$
b. $\Delta \mathrm{U}=\mathrm{Q}$
c. $\Delta \mathrm{U}=\mathrm{W}$
d. $W=Q$ 
15. Ana coloca en cubículos aislados térmicamente dos cuerpos de igual masa y diferentes temperaturas, como se indica en el gráfico. Del proceso transferencia de energía en forma de calor y de la temperatura cuando los sistemas alcanzan el equilibrio térmico se puede decir:
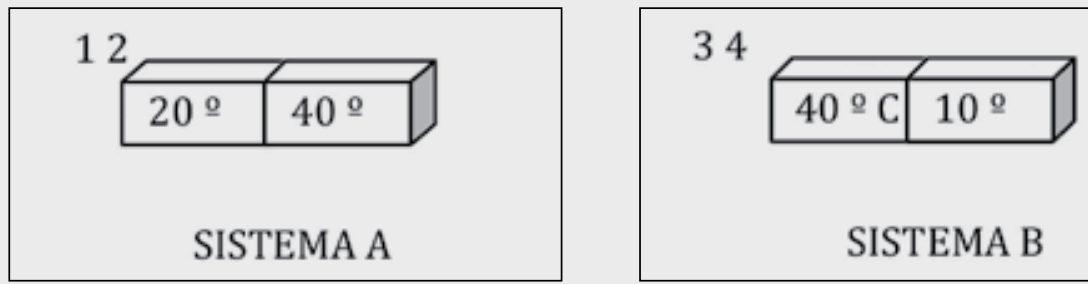

\section{SISTEMA B}

a. En el sistema A la energía fluye (calor) espontáneamente del bloque 1 al bloque 2. Después del equilibrio térmico ambos sistemas quedan con igual temperatura.

b. En el sistema A la energía fluye (calor) espontáneamente del bloque 2 al bloque 1. Después de llegar al equilibrio térmico la temperatura de $\mathrm{A}$ es mayor que la temperatura de $\mathrm{B}$.

c. En el sistema B la energía fluye (calor) espontáneamente de del bloque 3 al bloque 4. Después del equilibrio térmico la temperatura de ambos sistemas es la misma.

d. En el sistema B la energía fluye (calor) espontáneamente del bloque 4 al 3. Después del equilibrio térmico el sistema B queda a mayor temperatura que el sistema $\mathrm{A}$.

16. Una máquina termina es cualquier dispositivo que transforma la energía calorífica en trabajo. La gráfica del funcionamiento de una máquina que no viola la segunda ley de la termodinámica es: (tener en cuenta que $\mathrm{T}_{\mathrm{f}}$ es fuente fría, $\mathrm{T}_{\mathrm{c}}$ fuente caliente $\mathrm{y} \mathrm{W}$ es trabajo)

a.

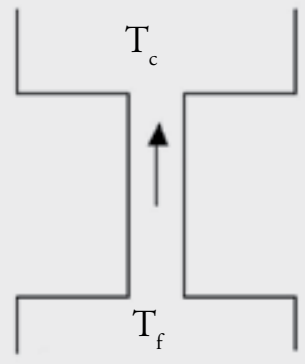

c.

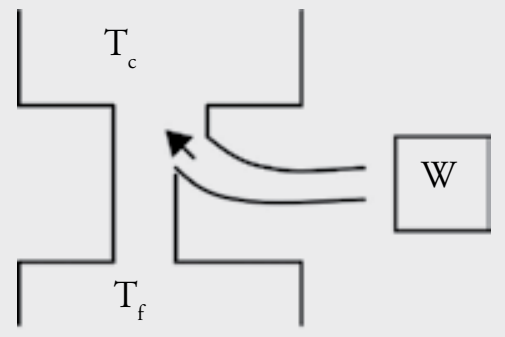

b.

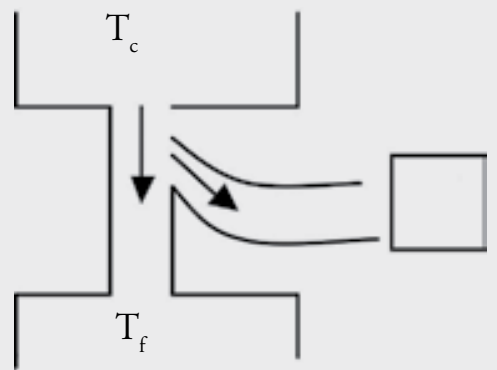

d.

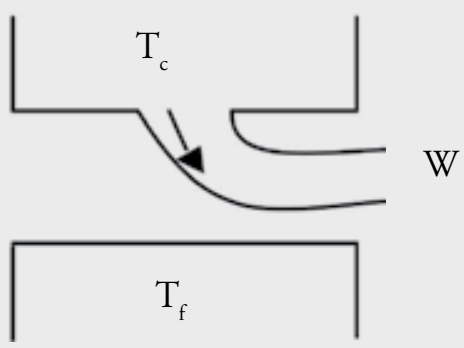

17. A partir del esquema de la estructura de la primera máquina de vapor para bombeo de agua (Newcomen, siglo XVIII), el proceso que se lleva cabo para su funcionamiento es:

a. El agua se evapora en la caldera. Se abre la llave A, el vapor sube y empuja el émbolo hacia arriba. Luego el émbolo es empujado manualmente hacia abajo lo que permite bombear el agua del pozo. El agua fría se utiliza como reserva para la caldera.

b. La llave B se abre, enfría el émbolo y este baja. De esta manera se bombea el agua del pozo. Luego se abre la llave A, el vapor sube y empuja el émbolo hacia arriba llevándolo a su posición inicial para un nuevo ciclo. 
c. La llave A se abre, sube el vapor y empuja el émbolo hacia arriba. Luego se cierra A y se abre B para que el resto de vapor se condense en el agua fría y el émbolo baje permitiendo así bombear el agua. De esta manera se inicia de nuevo el proceso.

d. No conozco la respuesta.

18. Un ingeniero dice haber construido una máquina ideal en la cual la energía no se degrada. Una manera de evidenciar si la máquina es ideal o no es constatar que:

a. Dicha máquina realiza más trabajo que las demás maquinas.

b. Dicha máquina trabaja más rápido que las además maquinas.

c. Dicha máquina no se calienta; su temperatura es la del ambiente

d. Dicha máquina se recalienta más que las demás maquinas.

$134<$ Universidad de San Buenaventura, Cali - Colombia 